\title{
Linking In-Game Events and Entities to Social Data on the Web
}

\author{
Owen Sacco, Maciej Dabrowski and John G. Breslin \\ Digital Enterprise Research Institute, \\ National University of Ireland, Galway, Ireland \\ owen.sacco@deri.org, maciej.dabrowski@deri.org and john.breslin@nuigalway.ie
}

\begin{abstract}
The Web of Data is an emerging term used to describe a recent trend for publishing and interlinking previously disconnected datasets on the Web in order to make them more valuable and useful as a whole. In the world of gaming, a parallel issue exists in that there are many different types of games, player profiles and web-based discussion systems for gamers that remain disconnected from each other due to a lack of standardised methods for expressing gaming entities and events. In this paper, we describe an approach to link entities in-game and gamer profiles to items on the Web, and vice versa, that exploits Semantic Web technologies. Previous work focused on client-side capturing of gamer information for online profiles, and in the reverse direction, creating game content from open data on the Web. Other work has tackled how community requirements can assist in altering games and providing updated content for gamers. Our work proposes a more generally-applicable solution that would provide for a set of terms commonly found in games to be used on the Web, and to list suitable Web information sources (e.g. Wikia games wikis) that could be used to enhance in-game play. This work will extends previous efforts to create games ontologies, resulting in a more structured RDF games ontology describing game types, features, events and character profiles. We describe how our ontology could be linked to existing Semantic Web vocabularies for describing social data on the Web, including Friend of a Friend (FOAF) and Semantically Interlinked Online Communities (SIOC).
\end{abstract}

Index Terms-Games, G2W, Ontology, Standards, Semantic Web, Social Data.

\section{INTRODUCTION}

Online gaming communities are dispersed over the Internet in the form of blogs, microblogs, forums, wikis, social networks and other social applications which are disconnected from one another. The datasets created by those communities all contain game information which are useful for gamers but are not easily discoverable. Moreover, gaming platforms allow gamers to publish their game accomplishments such as ingame achievements, trophies, events information, purchases, or rankings on the Web. This information is, however, isolated within the gaming platform or on the published site. The emerging Web of Data trend, where datasets are published in a standard form for easy interlinking, enables to view the whole Web as one massive integrated database. Nevertheless, game information is still not enriched with meta-structures that could be used both on the Web and also in games.

In this paper, we describe a model that provides a standard format for structuring gaming data across different online applications and gaming platforms such as Steam, PlayStation
Network (PSN), XBOX Live, Windows Live, Google Play, Apple Store, etc. The model describes various gaming features: gaming information, user profiles, character information, achievements, trophies, events (calendar events to massive online battles such as in Battlefield 3 and World of Warcraft), or chats. Among other applications, our model could be used: (1) to define meta-structures for characterising and representing game play abstractly that could then be re-used on the Web; (2) to integrate real-time events or other information from the Web within games. The latter could lead to a new kind of realtime game experience which also implies new game mechanics and rules. Furthermore, in this paper we provide an approach for defining fine-grained privacy preferences to the structured gaming data enabling gamers to control who can access their gaming information.

The remainder of this paper is structured as follows: next section discusses our model for structuring gaming data and also explains how this model maps to other, existing vocabularies. In section III we present how gaming information enriched with our model can be used on the Web and section IV presents how Web information can enhance game play. In section $\mathrm{V}$ we describe an approach for the creation of fine-grained access control preferences for controlling access to published game information. Section VI we provide some related work and section VII concludes the paper.

\section{MODEL}

In this section we present the Game2Web (G2W) ontology (see Figure 1): a vocabulary for describing information about the game, gamers and live events taking place during the game. We have identified the main concepts in online gaming communities as Persona, In-Game Event, Session, Achievement, Game and Transaction.

\section{A. Main Classes}

This section lists the main classes in the G2W ontology and describes their usage in more detail.

Persona: represents the gamer within a particular game. Hence, a gamer will have multiple personas, one (or more) for each game $\mathrm{s} / \mathrm{he}$ is playing. A persona is linked to a gamer through his/her online account, described as sioc: UserAccount which in turn is linked to the gamer's profile described as foaf:Person. A persona is based on a default game character and therefore the gamer's persona 


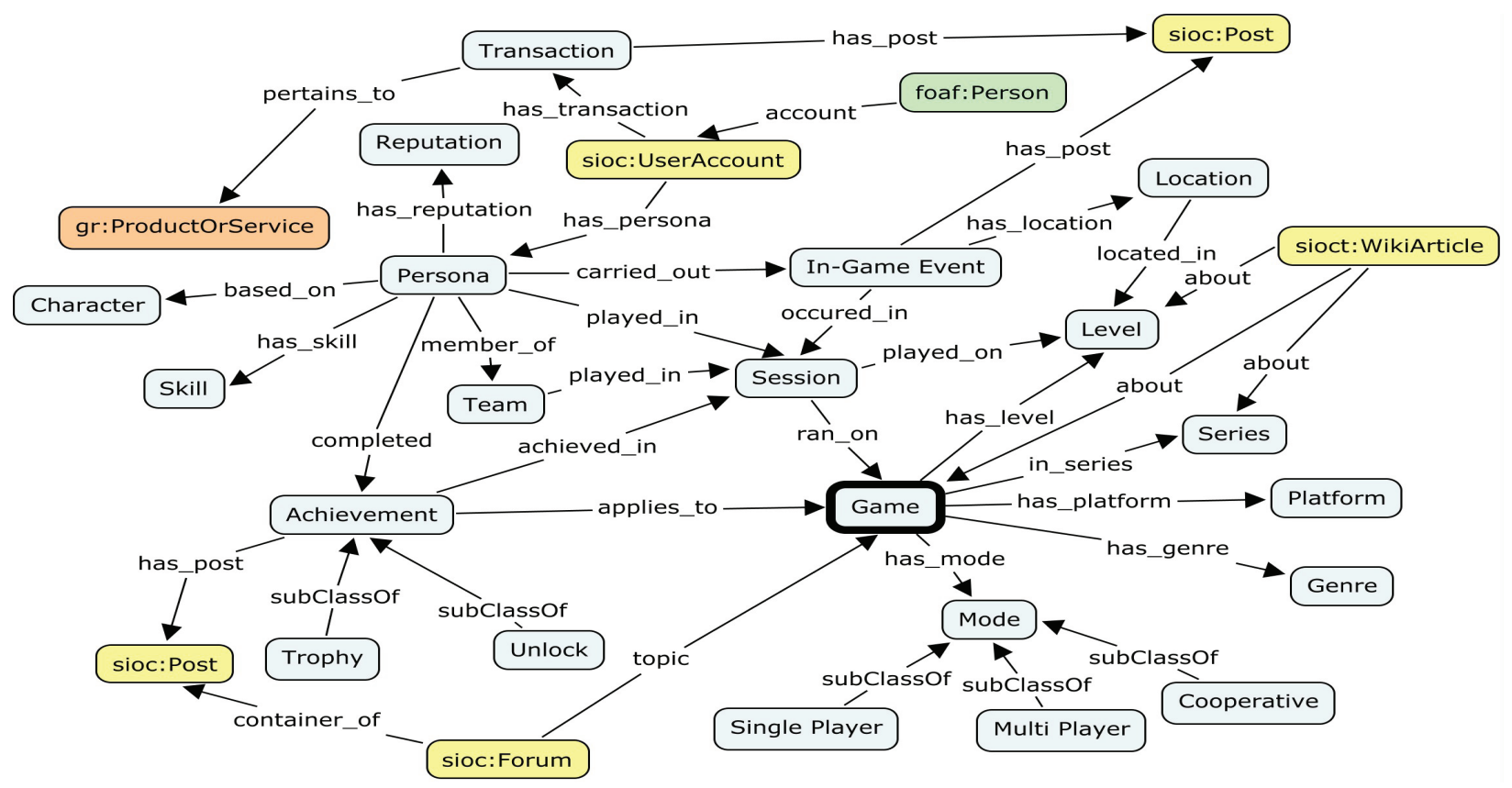

Fig. 1. A graphical representation of the Game2Web Ontology (G2W)

inherits the character's abilities, skills and any other characteristics; or a persona can be customised by the gamer (if allowed by the game). As the gamer progresses within the game, the gamer's persona might gain new skills or reputation and hence these are updated to the gamer's persona. A persona can also be part of a team or a clan, for instance in massively multiplayer online games (MMOG). During the game, a persona takes part in events; plays in particular sessions; and completes achievements.

In-Game Event: represents an event that happens during the game, for instance a particular task (for example to collect a treasure) that needs to be accomplished. Events occur during a particular session and also might occur in specific locations within the game's map. Once a gamer's persona completes major events, these accomplishments can be published on the Web as posts described using sioc:Post.

Session: is a particular moment in time during which a gamer is playing. A session might consist of a gamer playing a particular level of a game or a particular event when either a single gamer's persona or a team of gamers personas are playing.

Achievement: represents an accomplishment completed or gained by a gamer's persona. Examples of achievements include either earning trophies or unlocking items during a game. An achievement is bound to a particular game which is accomplished during a session, yet it can be posted on the Web as a sioc:Post on gaming forums (sioc:Forum).

Game: represents the game which a gamer is playing. A game can be characterised with various modes such as single player, multiplayer and/or cooperative game play (e.g. in Call of Duty where multiple gamers team up to complete the single player story together). A game can be part of a series (such as Assassin's Creed series); is of a particular genre (example Action-Adventure-Stealth game); runs on particle platforms (example PlayStation 3); contains levels; and also has wiki articles (such as game guides from Wikia) described using sioct:WikiArticle.

Transaction: refers to a gamer's purchases of games or ingame items (such as weapons) using the specific platform on which the game is running (example Google Play or PSN). This purchase can be published to the Web described as a sioc:Post.

\section{B. Describing Game Information using $G 2 W$}

The properties of the G2W ontology define the relationships amongst the classes. For instance, when a gamer is playing a game, this is described as a gamer's persona that carries out an in-game event or completes an achievement which occurs during a session in a particular level located in a specific level that runs on a particular game executed on a particular platform (example PlayStation 3) in a specific mode (example single player). All this information can be published on the Web as sioc:Post. The descriptions of these posts would use common entities: when referring to a particular game, the game's URI representing that game on the Web is used. This would make it easier for linking datasets together, for instance linking all the posts about a particular game that are stored in different blogging sites.

\section{Games to Web}

Publishing information to the Web in a standard format enables the information to be interlinked with other datasets that provides more information and can be seen as complete rather than as dispersed. Information published about a gamer through one platform can easily be interlinked with the 
gamer's information published from another platform. Hence, the gamer can keep track of all his/her achievements across multiple platforms. Moreover, standardisation of gaming information enables cross platform portability. For instance if an achievement is completed on one platform and the gamer decides to use another platform, the achievement will still count on the other platform without forcing the user to recomplete it. This also applies to the gamer's persona whereby the gamer can use the same persona on multiple platforms rather than having to accomplish the same tasks using a new persona from scratch on other platforms for the same game.

Apart from platform portability, structured game data can be enriched with additional data that provides a more complete view on gamers profile. For example, information about a completed achievement or a won battle in an online game can be linked to additional details (stored in other datasets), such as more details about the game level, the location of the map that can be pinpointed on an actual map of the game, the rules of the game etc. Such additional information can help other gamers in learning the details of the achievement, understanding its real value and the process of how the gamer completed it.

When publishing structured gaming data, gamers can compare their achievements and rankings amongst each other for the same games even though their data is stored in other datasets. Gamers can also benefit from systems that can recommend other players with similar rankings or who can be a suitable team mate; even those gamers that are playing on other servers. Most gaming forums can keep track of gamer's performance and game achievements but these are not interlinked with one another. Whilst having structured gaming data, players can then be paired even if their information reside in different datasets.

Games or gamers themselves can announce scheduled events that will occur in the future. Since the in-game information will be published using the G2W ontology, these announcements can be sent to gamers that are currently playing the game and that are eligible to play the event. These events can be added to the gamer's calendars which will remind the gamer of the event. Furthermore, in-game chats can be published and linked to other posts as sioc:Post or to social applications such as Facebook wall pages or as twitter streams.

\section{WEB TO GAMES}

The link between the Web and online gaming can be exploited not only from games to the Web but also through the linking of content and activities on the Web to the gaming world. Such inclusion of data from the Web that makes the gaming experience more realistic can take place in two contexts: (1) inclusion of digital real-world data, and (2) inclusion of Social Web data.

Inclusion of digital real-world data (e.g. recent news, real landscapes or historical events) in game environment is a practice used to increase the reality of scenarios and game play. Many flight simulators (e.g. Flight Simulator X [1] or Flight
Pro Sim [2]) include digital models of objects such as actual landscapes or airports and combine them with multiplayer mode to provide more realistic experience. This trend is also visible in some of the sports games, such as SSX from EA Sports, where the NASA topography was used for creation of snowboarding trails [11]. The next generations of games benefit from Augmented Reality such as DroidShooting [6] which bring game play into the gamers environment. The inclusion of historical events is especially visible in strategic games where players can participate in scenarios resembling actual campaigns and immerse in history (e.g Legends of War: Patton's Campaign or Medal of Honour series). Moreover, games which include historic information within the game, such as Assassin's Creed series, can easily be linked to other online historic information rather than relying only on in-game information which provides the gamer with a better gaming experience. Game guides from wikis (such as Wikia) can also be linked within games since these can be described as sioct:WikiArticle. Furthermore, pushing information from the Web into the game will also provide new game rules that will create a new gaming experience.

On the other hand, Social Network games propose to build game play on top of existing online communities and content. These connections are visible between the online social activities and formation of game clans that later lead to allies in game play. Another type of games exploit existing social platforms - The Sims Social [7] is a Facebook addition to the Sims series that utilises socialising features of Facebook to allow players to send and receive gifts in order to finish certain quests or objectives. In-game chat can also benefit from posts such as wall post on Facebook or microblog posts in twitter that can enhance game play.

\section{PRIVACY}

When publishing personal data on the Web, controlling access to this data is crucial in order not to end up being misused. The gamer must be provided with features that enable him/her to control how others can access and use their data, for example purchase information. Moreover, the access control privileges have to be dynamically enforced in such a way that the gamer is not hindered during the game with prompts whether the achievement should be published and to whom. Furthermore, the user must not maintain any user lists to whom s/he wants to publish the data but should be dynamically published to users based on specific attributes.

In this section we present an overview of the Privacy Preference Ontology (PPO) which provides the creation and enforcement of fine-grained access control policies on gaming data stored in multiple sources.

\section{A. The Privacy Preference Ontology (PPO)}

The Privacy Preference Ontology (PPO) [12] - http://vocab. deri.ie/ppo\# - is a light-weight Attribute-based Access Control (ABAC) vocabulary that allows people to describe fine-grained privacy preferences for restricting or granting access to specific Linked Data. Among other use-cases, PPO can be used to 
restrict part of a user's gaming records only to users that have specific attributes. It provides a machine-readable way to define settings such as "Grant read access to a particular game achievement only to those who are playing the same game" or "Grant read access to my gaming profile only to gamers having a similar or higher ranking" or "Grant read access to my purchase information to gamers who own the same game."

As PPO deals with $\mathrm{RDF}(\mathrm{S}) / \mathrm{OWL}$ data, a privacy preference, defines: (1) the resource, statement, named graph, dataset or context it must restrict access to; (2) the conditions refining what to restrict; (3) the access control type; and (4) a SPARQL query, (AccessSpace) i.e. a graph pattern representing what must be satisfied by the user requesting information. The access control type defines the Read and Write access control privileges (for reading or updating data).

The privacy preferences are enforced by the Privacy Preference Manager [13] which allows users to manage their privacy preferences and also grants or denies access to user's information when requested by others. Through the privacy preference manager, gamers can (1) authenticate to their instance and create privacy preferences for their gaming data; and (2) authenticate to other user's instance and access the filtered gaming data of these users. Moreover, the privacy preference manager can be used as an API by other Web applications to enforce gamer's privacy preferences for filtering their personal gaming information.

\section{RELATED WORK}

There is a link between the social interactions online and those within games: $76.2 \%$ of male and $74.7 \%$ of female players had made good friends within the game. This suggests that MMORPGs are highly socially interactive [8]. With this study, it enforces the importance of our model for publishing in-game events on Social platforms and also for interlinking different datasets together to provide more gaming information.

Other work [9] propose a concept of client side capturing of profile information within the working environment of a gamer playing online games and a profile server storing generic community-extendable gaming profile data models that can be individualised. This work also matches the gamer's profile to the user's online profile. Whereas this work focuses primarily on the profile, our work focuses on publishing ingame information that can be interlinked with other datasets that can enhance the gaming experience.

In order to enhance game play by using Web information, IBM's [10] online gaming architecture can be used to provide in-game services.

The GiantBomb forum [4] provides a service that extracts a user's information from various platforms, such as PlayStationNetwork (PSN) and XBOX Live. The information consists of all the games a person is playing on various platforms together with the characters, statistics, trophies, etc. In this forum you can browse a user's profile and find out what $\mathrm{s} / \mathrm{he}$ has been playing, and all the other information about their achievements. The service includes an API [5] that provides
XML / JSON data about the information which they collect from a user's gaming activities. However, the data is not enriched with meta-structures which makes it isolated from other datasets. Therefore, this data stored in this forum can be enriched using our model in order to take advantage of interlinking with other datasets and other Social data.

The Game Ontology Project (GOP) [3] is a framework for describing and analysing games. It provides a hierarchy of concepts abstracted from an analysis of many specific games. Although the aim of this project is to provide concepts about games, such concepts are incomplete and are not in a format that can be used to define in-depth game information for publishing and linking with other datasets on the Web.

\section{CONCLUSION}

In this paper we presented our Game2Web (G2W) ontology, a vocabulary for describing gaming information that can be published and interlinked with other datasets on the Web. It provides classes that describe gamer's personas, in-game events, game sessions, achievements, games and transactions. We have provided insights of how this gaming information can enhance the gaming experience by interlinking with other information on the Web and we have explained how gaming can be enhanced with information from the Web. We have also provided an overview of how the gamer can control access to his/her personal gaming information to those with similar attributes.

We plan to extend this model to provide in-depth game attributes for defining more information about the game, such as the rules. This would enable structured Web information to effect the game play in real-time that would provide a new gaming experience.

\section{REFERENCES}

[1] Flight Simulator X. https://www.microsoft.com/games/flightsimulatorx/.

[2] FlightProSim. http://flightprosim.com/.

[3] Game Ontology Project. http://www.gameontology.com.

[4] Giant Bomb. http://www.giantbomb.com/.

[5] Giant Bomb API. http://api.giantbomb.com/documentation/.

[6] Google Play Store: DroidShooting.

[7] The Sims Social. http://on.fb.me/QHswEM.

[8] H. Cole and M. D. Griffiths. Social interactions in massively multiplayer online role-playing gamers. In CyberPsychology and Behavior, August 2007.

[9] T. Hildebrandt, S. Bergsträßer, C. Rensing, and R. Steinmetz. Capturing and Storing Profile Information for Gamers Playing Multiplayer Online Games. In Proceedings of the 8th Annual Workshop on Network and Systems Support for Games (NetGames), NETGAMES2009.

[10] V. Megler. IBM: Online game infrastructures, Part 4: Address community requirements, game upgrades, and account maintenance. http: //www.ibm.com/developerworks/web/library/wa-games4/.

[11] K. Orland. Ars Technica: How NASA topography data brought dose of reality to SSX snowboarding courses.

[12] O. Sacco and A. Passant. A Privacy Preference Ontology (PPO) for Linked Data. In Proceedings of the Linked Data on the Web Workshop, LDOW2011.

[13] O. Sacco and A. Passant. A Privacy Preference Manager for the Social Semantic Web. In Proceedings of the 2nd Workshop on Semantic Personalized Information Management: Retrieval and Recommendation, SPIM2011, 2011. 